\title{
Emergence of Chaotic Scattering in Ultracold Er and Dy
}

\author{
T. Maier, ${ }^{1,}$ H. Kadau, ${ }^{1}$ M. Schmitt, ${ }^{1}$ M. Wenzel, ${ }^{1}$ I. Ferrier-Barbut, ${ }^{1}$ T. Pfau, ${ }^{1}$ A. Frisch,${ }^{2,3}$ S. Baier, ${ }^{2}$ K. Aikawa, ${ }^{2, \dagger}$ \\ L. Chomaz, ${ }^{2,3}$ M. J. Mark, ${ }^{2,3}$ F. Ferlaino, ${ }^{2,3}$ C. Makrides, ${ }^{4}$ E. Tiesinga,${ }^{5}$ A. Petrov, ${ }^{4,5}$ and S. Kotochigova ${ }^{4}$ \\ 15. Physikalisches Institut and Center for Integrated Quantum Science and Technology, \\ Universität Stuttgart, Pfaffenwaldring 57, 70569 Stuttgart, Germany \\ ${ }^{2}$ Institut für Experimentalphysik, Universität Innsbruck, Technikerstraße 25, 6020 Innsbruck, Austria \\ ${ }^{3}$ Institut für Quantenoptik und Quanteninformation, Österreichische Akademie der Wissenschaften, \\ 6020 Innsbruck, Austria \\ ${ }^{4}$ Department of Physics, Temple University, Philadelphia, Pennsylvania 19122, USA \\ ${ }^{5}$ Joint Quantum Institute and Center for Quantum Information and Computer Science, \\ National Institute of Standards and Technology and the University of Maryland, \\ 100 Bureau Drive, Stop 8423, Gaithersburg, Maryland 20899, USA \\ (Received 7 June 2015; revised manuscript received 21 September 2015; published 19 November 2015)
}

We show that for ultracold magnetic lanthanide atoms chaotic scattering emerges due to a combination of anisotropic interaction potentials and Zeeman coupling under an external magnetic field. This scattering is studied in a collaborative experimental and theoretical effort for both dysprosium and erbium. We present extensive atom-loss measurements of their dense magnetic Feshbach-resonance spectra, analyze their statistical properties, and compare to predictions from a random-matrix-theory-inspired model. Furthermore, theoretical coupled-channels simulations of the anisotropic molecular Hamiltonian at zero magnetic field show that weakly bound, near threshold diatomic levels form overlapping, uncoupled chaotic series that when combined are randomly distributed. The Zeeman interaction shifts and couples these levels, leading to a Feshbach spectrum of zero-energy bound states with nearest-neighbor spacings that changes from randomly to chaotically distributed for increasing magnetic field. Finally, we show that the extreme temperature sensitivity of a small, but sizable fraction of the resonances in the Dy and Er atom-loss spectra is due to resonant nonzero partial-wave collisions. Our threshold analysis for these resonances indicates a large collision-energy dependence of the three-body recombination rate.

DOI: 10.1103/PhysRevX.5.041029

\section{INTRODUCTION}

Anisotropic interactions are a central and modern tool for engineering quantum few- and many-body processes [1]. A prominent example of such an interaction is the long-range dipole-dipole interaction (DDI) acting, for instance, between polar molecules [2], Rydberg atoms [3], or magnetic atoms [4]. Over the years, fascinating quantum effects of the anisotropy have been observed, such as the $d$-wave collapse of a dipolar Bose-Einstein condensate [5],

\footnotetext{
*Corresponding author. t.maier@physik.uni-stuttgart.de

${ }^{\dagger}$ Present address: Department of Physics, Graduate School of Science and Engineering, Tokyo Institute of Technology, Meguro-ku, Tokyo 152-8550, Japan.

"Also at: NRC "Kurchatov Institute" PNPI 188300, Division of Quantum Mechanics, St. Petersburg State University, 198904, Russia.

Published by the American Physical Society under the terms of the Creative Commons Attribution 3.0 License. Further distribution of this work must maintain attribution to the author(s) and the published article's title, journal citation, and DOI.
}

Subject Areas: Atomic and Molecular Physics, Quantum Physics the deformation of the Fermi surface [6], and the control of stereodynamics in dipolar collisions [7]. Moreover, the DDI is expected to give rise to a plethora of few- and manybody phenomena, which still await observation, such as universal few-body physics [8,9], rotonic features [10,11], two-dimensional stable solitons [12], and the supersolid phase [13].

Recently, atomic species in the lanthanide family became available to the field of ultracold quantum gases. The interaction between magnetic lanthanide atoms, such as $\mathrm{Er}$ $[14,15]$ and Dy [16,17], is highly anisotropic. This is not only due to the long-range DDI, originating from their large magnetic moment, but also to the shorter-ranged van der Waals interaction [18], which exhibits anisotropic contributions arising from the large orbital angular momentum of their valence electrons.

For magnetic lanthanides, which also include the successfully laser-cooled elements Ho [19] and Tm [20], the orbital anisotropy is a consequence of a partially filled submerged $4 f$ electron shell that underlies a closed outer $6 s$ shell. This leads to an electronic ground state with a total atomic angular momentum $\vec{j}$ with $j \gg 1$. Consequently, in 
collisions between such atoms there exist $(j+1)^{2}$ nondegenerate (gerade) molecular Born-Oppenheimer (BO) potentials and a correspondingly large manifold of collision channels with associated molecular bound states. This is in sharp contrast to the one or two BO potentials encountered in alkaline-earth and alkali-metal atom collisions. In addition, the anisotropy or orientation dependence of the BO potentials strongly mixes collision channels with large relative orbital angular momentum $\vec{\ell}$ between the atoms even for our ultracold collisions with a $\ell=0$, $s$-wave initial channel. The complexity of the molecular forces are reflected in a dense spectrum of Fano-Feshbach resonances as a function of magnetic field $B$, as recently observed in Er [14,21] and Dy [22]. In Er a statistical analysis of the spacings between resonances has shown correlations that revealed chaotic scattering. The data set of the initial Dy experiments was too small to extract statistically significant correlations.

Chaotic behavior is manifest in a variety of complex systems ranging from atomic to nuclear and solid-state physics. In atomic physics, chaos was originally studied with Rydberg states of $\mathrm{H}$ and $\mathrm{He}$ in a magnetic field [23]. Later on, a variety of more complex atoms and ions in highly excited states showed signatures of chaotic spectral distributions [24]. The origin of chaos in these systems was traced back to a strong mixing of many-electron excited states by the Coulomb interaction [25]. A chaotic level distribution is also common in a variety of solid-state systems ranging from those with strong many-body interactions to the motion of particles in irregular potentials [26,27]. Experiments in nuclear physics [28,29] have also produced substantial evidence for chaotic neutron resonance spectrum fluctuations, which agree with predictions of random matrix theory (RMT). Similar agreement was found from numerical simulations based on nuclear shell models [30,31]. Moreover, Refs. [32,33] suggested that chaos is a generic property of nuclei with multiple degrees of freedom (i.e., multiple active shells), which become completely mixed.

This article describes a joint effort to understand ultracold scattering and Fano-Feshbach spectra of strongly magnetic Er and Dy atoms. In particular, we report on the measurement and statistical analysis of Fano-Feshbach spectra for Dy and Er between $B=0$ and $70 \mathrm{G}$ at gas temperatures $T$ below and around $1 \mu \mathrm{K}$. Here, $1 \mathrm{G}=0.1 \mathrm{mT}$. We observe that both elements have similar chaotic scattering. We present a RMT-inspired model to gain insight into their statistical properties as well as theoretical evidence based on coupled-channels calculations with a microscopic Hamiltonian that chaotic scattering requires both strong molecular anisotropy and Zeeman mixing to fully develop. Limitations of the RMT are also discussed. Finally, we present experimental data and a comparison to a resonant trimer model to show that our increase in resonance density with temperature is a consequence of the strong collision-energy dependence of transitions from entrance $d$-wave channels of three free atoms to resonant trimer states.

\section{EXPERIMENT}

\section{A. Measurement}

The experimental study of Fano-Feshbach resonances in Er and Dy is based on high-resolution trap-loss spectroscopy on spin-polarized thermal samples. Ultracold bosonic ${ }^{164}$ Dy samples are created by direct loading from a narrowline magneto-optical trap, operating on the $626 \mathrm{~nm}$ cycling transition, into a single-beam optical dipole trap (ODT) [34]. By moving the last focusing lens of the ODT, the atoms are transported from the magneto-optical trap chamber to the science cell. The ODT is created with a $100 \mathrm{~W}$ fiber laser at a wavelength of $1070 \mathrm{~nm}$. We achieve a transport efficiency close to unity. This fiber laser, however, causes atom loss due to its longitudinal multimode structure [35]. Therefore, we transfer the atoms into a second single-beam ODT, created by a $55 \mathrm{~W}$ solid-state laser at a wavelength of $1064 \mathrm{~nm}$. Finally, forced evaporative cooling in a crossed ODT leads to a sample of $10^{5}$ atoms in the energetically lowest Zeeman sublevel, $m_{j}=-8$ at $T=600 \mathrm{nK}$.

High-resolution trap-loss spectroscopy is performed on a spin-polarized bosonic ${ }^{168} \mathrm{Er}$ sample at $T=1400 \mathrm{nK}$ and compares this spectrum with that obtained at a 4 times lower temperature measured in previous work for ${ }^{168} \mathrm{Er}$ as well as for fermionic ${ }^{167} \mathrm{Er}$ [21]. The experimental procedures for creating bosonic and fermionic samples are described in Refs. [14,15], respectively. Bosons (fermions) are prepared in the lowest Zeeman sublevel, $m_{j}=-6$ $\left(m_{f}=-19 / 2\right)$. Erbium samples are trapped in a crossed ODT and contain about $10^{5}$ atoms.

\section{B. Feshbach spectroscopy}

Feshbach spectroscopy is performed in a similar manner for the two species. The magnetic field is ramped up over a few milliseconds to a magnetic-field value $B$, where the atoms are held in the ODT for $500 \mathrm{~ms}$ for Dy, $400 \mathrm{~ms}$ for ${ }^{168} \mathrm{Er}$, and $100 \mathrm{~ms}$ for ${ }^{167} \mathrm{Er}$. During this time, inelastic three-body recombination causes atom loss from the ODT. At resonance, the recombination process is enhanced because of the coupling between the atomic-threshold state and a molecular state leading to a resonant increase of the atom loss. We identify the field locations of maximum loss as the positions of Fano-Feshbach resonances [36]. The atom number is probed by standard timeof-flight absorption imaging at low magnetic field. We record atom-loss features for magnetic-field values between 0 and $70 \mathrm{G}$ in steps of a few $\mathrm{mG}$. Figure 1(a) shows the normalized loss spectrum for the ${ }^{164}$ Dy isotope, where we identify 309 resonances. For ${ }^{168} \mathrm{Er}$ at $T=1.4 \mu \mathrm{K}$, there are 

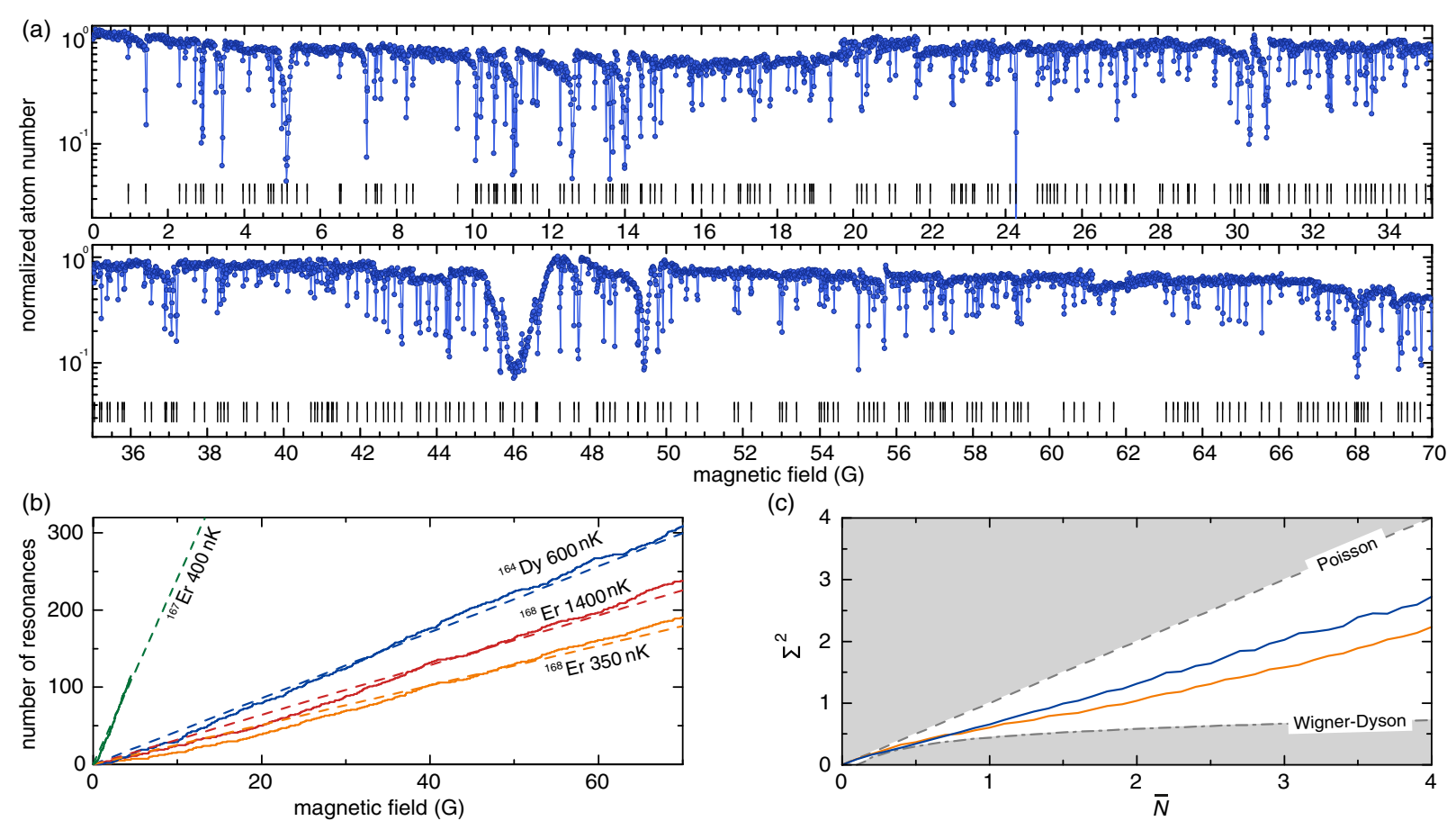

FIG. 1. (a) Trap-loss spectroscopy mapping of the Fano-Feshbach spectrum of ${ }^{164}$ Dy as a function of magnetic field between $B=0$ and $70 \mathrm{G}$ with a data point every $14.5 \mathrm{mG}$ and temperature $T=600 \mathrm{nK}$. Each data point is an average of three measurements. (b) Staircase function for the number of resonances as a function of $B$ for ${ }^{164} \mathrm{Dy},{ }^{168} \mathrm{Er}$ at two temperatures, and fermionic ${ }^{167} \mathrm{Er}$. Dashed lines are linear fits forced to pass through the origin. Their slopes give a mean density of resonances of $\bar{\rho}=4.3 \mathrm{G}^{-1}$ for ${ }^{164} \mathrm{Dy}$, $2.7 \mathrm{G}^{-1}$ for ${ }^{168} \mathrm{Er}$ at $T=350 \mathrm{nK}, 3.4 \mathrm{G}^{-1}$ for $\mathrm{Er}$ at $T=1.4 \mu \mathrm{K}$, and $25.6 \mathrm{G}^{-1}$ for ${ }^{167} \mathrm{Er}$. (c) Number variance of the experimental data as a function of scaled $B$-field interval $\bar{N}=\Delta B \bar{\rho}$. The experimental data for ${ }^{164}$ Dy (blue line) and ${ }^{168} \mathrm{Er}$ at $T=350 \mathrm{nK}$ (orange line) lie between the variances for an uncorrelated Poisson distribution (dashed line) and the correlated Wigner-Dyson distribution (dot-dashed line).

238 resonances. The Fano-Feshbach scan of fermionic ${ }^{167} \mathrm{Er}$ is carried out from 0 to $4.4 \mathrm{G}$ and yields 115 resonances.

The understanding of the richness of the scattering in Er and Dy requires the development of sophisticated microscopic coupled-channels scattering models. We defer such analysis until later in this paper and first analyze our data following the statistical approach based on the RMT advocated by Ref. [21]. In particular, we study the correlations between resonance locations via the nearestneighbor spacing (NNS) distribution and set up a RMT-like model, which accounts for the structure of our $B$-dependent microscopic Hamiltonian, to get intuition about these NNSs. In our description of the coupled-channels calculations, limitations of such a RMT-like model are discussed.

\section{Statistical analyses}

Our statistical analysis starts with the construction of the staircase function, which is a steplike function that counts the number of resonances below magnetic-field value $B$ [37]. Figure 1(b) shows the staircase function for Dy and Er. For both species the function is well fit by a linear curve forced to pass through the origin. Its slope $\bar{\rho}$ corresponds to the density of resonances. Deviations below and above the fit occur for small and large $B$, respectively. The fitted resonance densities are given in the caption. Remarkably, the density of resonances of ${ }^{168} \mathrm{Er}$ at $T=1.4 \mu \mathrm{K}$ is $25 \%$ higher than the one observed at $350 \mathrm{nK}$. The discussion of the origin of this sensitivity is postponed until Sec. V. The density $\bar{\rho}$ for bosonic Dy is $50 \%$ larger than for bosonic Er. This is caused by the larger $\vec{j}$ of Dy and, thus, its larger number of allowed collision channels. The much larger density $\bar{\rho}$ of $25.6 \mathrm{G}^{-1}$ for the fermionic ${ }^{167} \mathrm{Er}$ is due to its additional hyperfine structure.

Fluctuations in the number of resonances within a magnetic-field interval $\Delta B$ is a second measure of the statistical properties of the spacings between resonances. Formally, it is defined as the dimensionless number variance $\Sigma^{2}=\overline{N^{2}}-\bar{N}^{2}$, where $\bar{N}=\sum_{i=0}^{M-1} N_{i} / M, \overline{N^{2}}=$ $\sum_{i=0}^{M-1} N_{i}^{2} / M$, and $N_{i}$ is the number of resonances in the field interval $[i \Delta B,(i+1) \Delta B]$, with $i=0, \ldots, M-1$, such that $M \Delta B=B_{\max }$ and $B_{\max }=70 \mathrm{G}$ for both species. Consequently, $\bar{N} \equiv \Delta B \bar{\rho}$. For shot noise or a Poissonian distribution, we expect $\Sigma^{2}=\bar{N}$. Figure 1(c) compares $\Sigma^{2}$ for our Dy and Er data as a function of $\bar{N}$. The fluctuations for both species monotonically increase with $\Delta B$ but are 
substantially less than the shot-noise limit. While this behavior was previously demonstrated for $\mathrm{Er}$ [21], the present results provide the first evidence of correlation in Dy and indicate similarity between the species.

These correlations between resonance locations are further studied using the nearest-neighbor spacings distribution $P(s)$, where $s=\delta B \bar{\rho}$ and $\delta B$ is the field spacing between two adjacent resonances in the spectrum. Figures 2(a) and 2(b) show the computed NNS distribution of our experimental data, derived from the number of NNS, $S_{i}$, with spacings $s$ between $i \delta s$ and $(i+1) \delta s$, where $i=0,1, \ldots$ and $\delta s \approx 0.3$. The NNS distributions have clear deviations from both the Poisson $P_{P}(s)=\exp (-s)$ and Wigner-Dyson $P_{\mathrm{WD}}(s)=(\pi / 2) s \exp \left[-(\pi / 4) s^{2}\right]$ distribution, two well-known distributions within RMT [21]. A Poisson distribution corresponds to a random distribution of resonance locations, while a Wigner-Dyson distribution corresponds to a situation where neighboring resonances "avoid" each other and $P_{\mathrm{WD}}(s) \propto s$ for $s \rightarrow 0$. Deviations are also seen in Fig. 1(c), where for both atomic species the variance $\Sigma^{2}$ does not agree with the corresponding predictions for these distributions. The experimental NNS distributions in Figs. 2(a) and 2(b) have also been fit to the Brody distribution $P_{B}(s, \eta)=b(1+\eta) s^{\eta} \exp \left[-b s^{\eta+1}\right]$, an
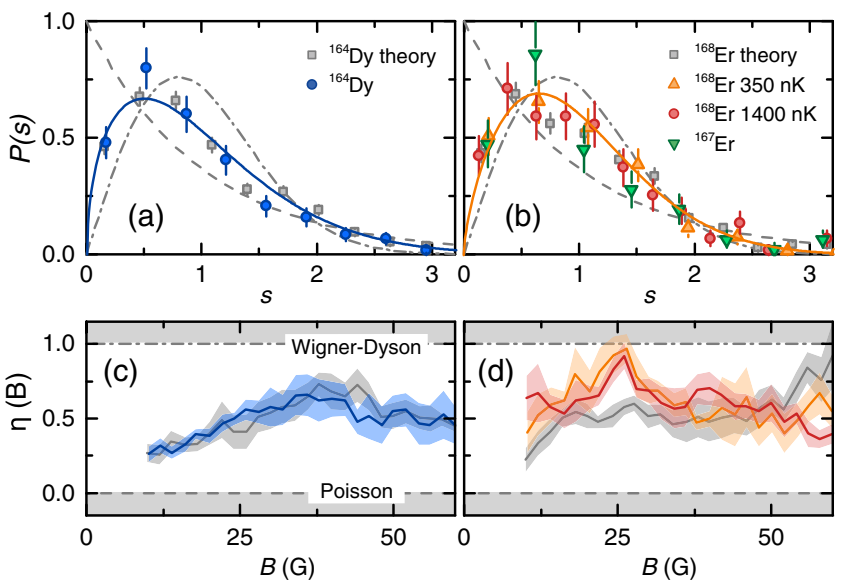

FIG. 2. (a) Nearest-neighbor spacing distribution $P(s)$ determined from all observed Fano-Feshbach resonances for ${ }^{164} \mathrm{Dy}$ (blue markers). Dashed and dot-dashed curves are the Poisson and Wigner-Dyson distribution, respectively. The solid line is a Brody distribution with $\eta=0.45$ (7) fit to the experimental data. (b) Distributions $P(s)$ for ${ }^{168} \mathrm{Er}$ at $T=350 \mathrm{nK}$ and $T=1.4 \mu \mathrm{K}$ (orange and red markers, respectively), and ${ }^{167} \mathrm{Er}$ at $T=0.4 T_{F}$ (green markers), where $T_{F}$ is the Fermi temperature of the gas. The solid line is a Brody distribution with $\eta=0.68(9)$ fit to the ${ }^{168} \mathrm{Er}$ data at $T=350 \mathrm{nK}$. Panels (c) and (d) show the magneticfield-resolved Brody parameter $\eta(B)$ as a function of magnetic field for ${ }^{164} \mathrm{Dy}$ and ${ }^{168} \mathrm{Er}$, respectively. The Brody parameters for the Poisson and Wigner-Dyson distribution are 0 and 1 , respectively. Gray markers and lines in all four panels are results from our coupled-channels calculations. The $1 \sigma$ error bars in (a) and (b) correspond to Poisson counting errors, while shaded bands in (c) and (d) are $1 \sigma$ statistical uncertainties of the fits to the data. empirical function that interpolates between $P_{P}(s)$ and $P_{\mathrm{WD}}(s)$ for $\eta=0$ and 1 , respectively, and $b$ is a normalization constant [38]. The values for $\eta$ reported in the caption indicate intermediate or mixed behavior of the data.

We present the magnetic-field resolved Brody parameter $\eta(B)$ in Figs. 2(c) and 2(d) obtained from a fit to the NNS distribution of resonances located in moving intervals $[B-\Delta B / 2, B+\Delta B / 2]$, with $\Delta B=20 \mathrm{G}$. It has a nonnegligible $1 \sigma$ uncertainty equally limited by the quality of the fit and the number of Feshbach resonances in an interval or bin. The latter uncertainty is reflected in the bin-to-bin variation of $\eta(B)$. For Dy we observe that $\eta$ increase linearly with field for small $B$, which saturates at a value of $\approx 0.5$ for $B>30 \mathrm{G}$. For Er the Brody parameter fluctuates around 0.5. Interestingly, the Er data at our two temperatures have a similar behavior, indicating that the larger density of resonances at higher $T$ does not impact the degree of correlation between their spacings.

\section{RMT ENSEMBLE MODEL}

Random matrix theory is based on the powerful notion that the statistics of eigenvalues and eigenfunctions of a complex system can be studied by replacing the microscopic Hamiltonian by an ensemble of random Hamiltonians. In this spirit, we construct a RMT-inspired model for weakly bound molecular dimer states to test the distribution of Fano-Feshbach resonances.

Our RMT model is based on the statistics of eigenvalues of the $N \times N$ real, symmetric matrix $H_{\mathrm{RMT}}=H_{0}+H_{Z}$, where matrices $H_{0}$ and $H_{Z}$ represent the $B=0$ Hamiltonian and the Zeeman interaction of the two atoms, respectively. Without loss of generality we can assume that $H_{Z}$ is a diagonal matrix with matrix elements given by $m g \mu_{B} B$, where $m$ is an integer between $-2 j$ and $2 j$, corresponding to the sum of the projection quantum numbers of the atomic angular momenta, $g$ is the atomic Landé factor, and $\mu_{B}$ is the Bohr magneton. The Zeeman interaction does not depend on the rotational state of the molecule and, thus, entries in $H_{Z}$ correspond to states with a definite value for $\ell$ and its projection. $H_{0}$ is then the $B=0$ Hamiltonian expressed in this basis. It is also convenient to define $H_{0}=H_{d}+H_{\mathrm{cpl}}$, where diagonal matrix $H_{d}$ contains the diagonal matrix elements of $H_{0}$ and $H_{\mathrm{cpl}}$ is the matrix of all its off-diagonal elements. The eigenvalues of $H_{d}$ can then be interpreted as the energies of rovibrational levels of the isotropic contribution of the molecular BO potentials, while $H_{\mathrm{cpl}}$ describes mixing due to the anisotropic contributions of these potentials.

We generate members of our ensemble of $H_{\mathrm{RMT}}$ by choosing random matrix elements for $H_{Z}, H_{d}$, and $H_{\mathrm{cpl}}$ based on specific distributions. The values of $m$ in $H_{Z}$ are uniformly distributed integers between $-2 j$ and $2 j$. The matrix elements of $H_{d}$ are chosen according to a Brody distribution with variable Brody parameter $\eta_{d} \in[0,1]$ and 
with a mean energy spacing between bound states $\epsilon_{d}$. Finally, matrix elements of $H_{\mathrm{cpl}}$ are chosen as Gaussiandistributed real numbers with zero mean and standard deviation $\nu_{\mathrm{cpl}}$, thereby on average coupling all diagonal elements equally. Notice that this construction deviates from that for a true Gaussian orthogonal ensemble, where all matrix elements of a symmetric Hamiltonian are Gaussian distributed [39].

We apply the RMT model to the case of ${ }^{168} \mathrm{Er}$. The relevant species-specific quantities are $j=6, g=1.16$, and $\epsilon_{d}$ is chosen to roughly reproduce the observed density of Fano-Feshbach resonances of ${ }^{168} \mathrm{Er}$ and is set to $\epsilon_{d} / h=6.4 \mathrm{MHz}$, where $h$ is Planck's constant. Figure 3(a)
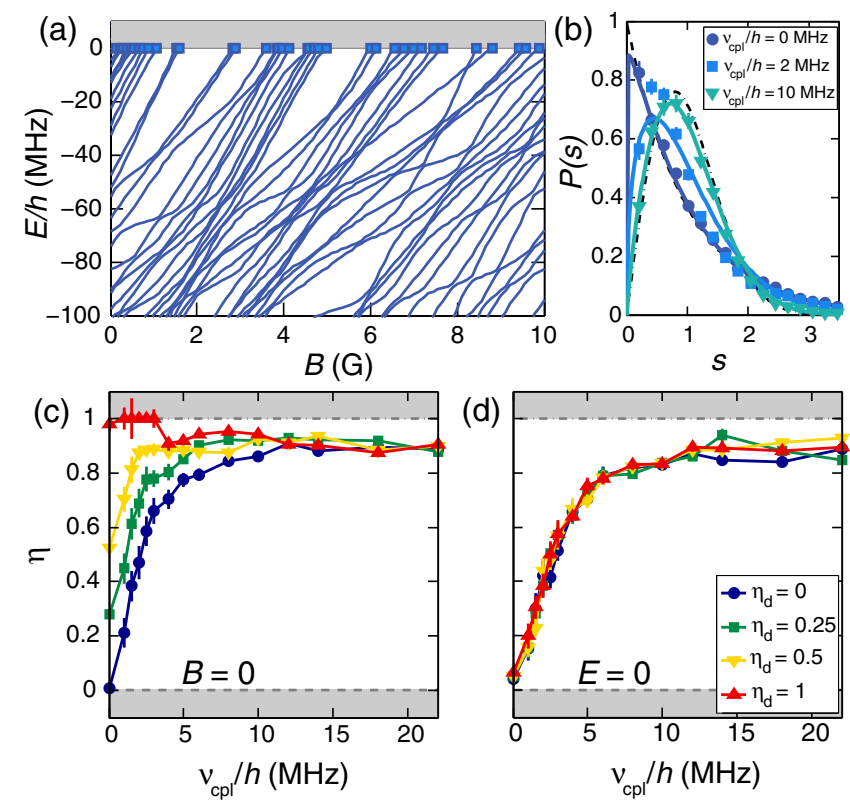

FIG. 3. Molecular spectrum and NNS distributions of FanoFeshbach resonances of ${ }^{168} \mathrm{Er}$ calculated from our RMT model. (a) Example of a spectrum of molecular binding energies as a function of $B$ for $\nu_{\mathrm{cpl}} / h=2 \mathrm{MHz}, \eta_{d}=0$. Here, $h$ is Planck's constant. Squares at $E / h=0 \mathrm{MHz}$ indicate crossings of molecular levels with the threshold of two $m_{j}=-6$ atoms and correspond to the position of Feshbach resonances. For the sake of visibility, we show only the spectrum between $B=0$ and $10 \mathrm{G}$. (b) NNS distributions of simulated Feshbach resonances for $\eta_{d}=0$ and $\nu_{\text {cpl }} / h=0 \mathrm{MHz}$ (circles), $2 \mathrm{MHz}$ (squares), and $10 \mathrm{MHz}$ (triangles). The dashed and dash-dotted lines are Poisson and Wigner-Dyson distributions, respectively. Solid lines are best-fit Brody distributions with $\eta=0.03(1), \eta=0.41(5)$, and $\eta=0.82$ (1) for $\nu_{\text {cpl }} / h=0,2$, and $10 \mathrm{MHz}$, respectively. (c) Fitted Brody parameters of the nearest-neighbor energy-spacing distribution of the eigenvalues of $H_{\mathrm{RMT}}$ at $B=0 \mathrm{G}$ as a function of $\nu_{\mathrm{cpl}}$. Circles, squares, down triangles, and up triangles correspond to $\eta_{d}=0,0.25,0.5$, and 1, respectively. (d) Fitted Brody parameters of the NNS distribution of the Feshbach resonances as a function of $\nu_{\mathrm{cpl}}$ and for four $\eta_{d}$ using the same marker code as in (c). In panels (b)-(d) distributions are obtained by averaging over 15 realizations of $H_{\mathrm{RMT}}$, each of dimension $500 \times 500$ and using Feshbach resonances computed up to $B=85 \mathrm{G}$. shows an example of a molecular spectrum, the eigenvalues of $H_{\mathrm{RMT}}$ obtained with our RMT model as a function of $B$ with $\eta_{d}=0$ and $\nu_{\mathrm{cpl}} / h=2 \mathrm{MHz}$. We observe that as $B$ increases, weakly bound molecular states avoid each other multiple times before reaching the two-atom threshold creating a Feshbach resonance. When we turn off $H_{\mathrm{cpl}}$, the levels cross. Similar $B$-field dependencies of the eigenvalues occur for $\eta_{d}>0$.

We investigate the effect of the parameters $\nu_{\mathrm{cpl}}$ and $\eta_{d}$ on the NNS distribution of the Fano-Feshbach resonances as well as that of the $B=0$ molecular levels. Figure 3(b) shows the NNS distribution of Feshbach resonances, obtained by averaging over 15 realizations of $H_{\mathrm{RMT}}$, for four values of $\nu_{\mathrm{cpl}}$ and $\eta_{d}=0$. For negligible $\nu_{\mathrm{cpl}}$, the distribution follows $P_{P}(s)$ and approaches $P_{\mathrm{WD}}(s)$ when the anisotropic coupling strength $\nu_{\mathrm{cpl}}$ is large compared to $\epsilon_{d}$. In fact, we find that a larger $\epsilon_{d}$ requires a larger $\nu_{\mathrm{cpl}}$ to develop correlations.

Figures 3(c) and 3(d) show Brody parameters fit to NNS distributions as functions of $\nu_{\mathrm{cpl}}$ and $\eta_{d}$. Figure 3(c) shows $\eta$ for the $B=0$ molecular binding energies. For $\nu_{\mathrm{cpl}}=0$, the Brody parameter is simply $\eta_{d}$, as expected from the distribution of the diagonal $H_{d}$, while for larger interaction anisotropy $\nu_{\text {cpl }}$, the parameter $\eta \approx 0.9$, close to a WignerDyson distribution, independent of $\eta_{d}$. Figure 3(d) shows $\eta$ extracted from the RMT Feshbach resonance locations as a function of $\nu_{\mathrm{cpl}}$. It suggests that the correlation in the NNS of the resonances is caused by $\nu_{\text {cpp }}$, whereas it appears fairly independent of $\eta_{d}$. More precisely, the Brody parameter fitted to these distributions rapidly increases from $\eta \approx 0$ to $\eta \approx 0.8$ for $\nu_{\text {cpl }} \lesssim \epsilon_{d}$ and tends to one for larger $\nu_{\text {cpl }}$. We conclude from the RMT model that the correlations between the locations of the Fano-Feshbach resonance are essentially due to the avoided crossings between weakly bound molecular states at finite $B$ and are only weakly dependent on the energy distribution at $B=0$. In fact, these correlations increase for increasing $\nu_{\mathrm{cpl}}$.

\section{MICROSCOPIC COUPLED-CHANNELS MODEL}

\section{A. Realistic setup}

A quantitative understanding of the origin of the chaotic resonance distribution requires coupled-channels and bound-state calculations with physically realistic angular-momentum couplings and interaction potentials. We do so here based on the time-reversal symmetric Hamiltonian for the relative motion of Dy and Er described in Refs. [21,40]. It contains the Zeeman Hamiltonian, the molecular vibration and rotation, and the molecular interactions with isotropic (orientation-independent) and anisotropic (orientation-dependent) contributions, $\hat{V}_{i}(R)$ and $\hat{V}_{a}(\vec{R})$, respectively, where $\vec{R}$ describes the separation $R$ and orientation of the atom pair $\hat{R}$. The potential has eight 
tensor operators coupling the two atomic and relative orbital angular momenta, $\vec{\jmath}_{1}, \vec{J}_{2}$, and $\vec{\ell}$. For $B=0$, the total angular momentum $\vec{J}=\vec{J}_{1}+\vec{\jmath}_{2}+\vec{\ell}$ is conserved. For $B>0 \mathrm{G}$, only the projection $M$ of $\vec{J}$ along $\vec{B}$ is conserved. The zero of energy of the Hamiltonian is the energy of an atom pair in the absolute lowest Zeeman sublevel, $m_{j \alpha}=-j_{\alpha}$.

The potentials $\hat{V}_{i}(R)$ and $\hat{V}_{a}(\vec{R})$ contain short-ranged exchange, medium-ranged van der Waals, as well as long-range magnetic dipole-dipole interactions. We use the isotropic van der Waals coefficient $C_{6}=1723 E_{h} a_{0}^{6}$ and anisotropic coefficients spread over $\Delta C_{6}=174 E_{h} a_{0}^{6}$ for $\mathrm{Er}$ [21]. For Dy we have improved the value of van der Waals coefficients of Ref. [40] by including additional experimental and theoretical transition frequencies and oscillator strengths [41-44] and now use $C_{6}=2003 E_{h} a_{0}^{6}$ and spread $\Delta C_{6}=188 E_{h} a_{0}^{6}$. In particular, the anisotropic spread for Dy has significantly increased. Here, $E_{h}=4.360 \times 10^{-18} \mathrm{~J}$ is the Hartree energy and $a_{0}=0.05297 \mathrm{~nm}$ is the Bohr radius.

\section{B. Bound-state calculations}

In Ref. [21] we performed initial coupled-channels calculations of the scattering between ultracold Er atoms and predicted that tens of partial waves $\ell$ should have been included as the strength of the anisotropic contribution is large. We, however, were unable to reach numerical convergence with respect to the number of coupled equations.

Here, we circumvent this limitation by performing multichannel bound-state calculations, in which we use $B=0$ eigenstates as a basis for those at $B>0 \mathrm{G}$. For $B=0$, where $J$ is a good quantum number, at most 49 and 81 Bose-symmetrized and parity-conserving channels are coupled for Er and Dy, respectively. The $B=0$ coupled Schrödinger equations are discretized on the interval $R \in\left[0, R_{\max }\right]$ assuming zero boundary conditions and solved as a matrix eigenvalue problem [45-48]. For each $J$, only eigenstates with energies between $\left[E_{0}, E_{1}\right]$ surrounding the zero of energy are computed and stored. The bound states for $B>0 \mathrm{G}$ are solutions of the matrix eigenvalue problem that includes all computed $B=0$ solutions with $|M| \leq J \leq J_{\max }$ and their coupling due to the Zeeman interaction. Selection rules of the Zeeman interaction ensure that there only exists direct coupling between $J$ and $J^{\prime}$ zero-field eigenstates with $J-J^{\prime}=0$, \pm 1 . For both species, $R_{\max }=1000 a_{0}, E_{0} / h=-3 \mathrm{GHz}$, and $E_{1} / h=0.9 \mathrm{GHz}$, ensuring that Feshbach resonance locations below $70 \mathrm{G}$ are converged.

In this section on the microscopic calculations we focus on analyzing the spectra at our coldest temperatures, where the initial collision channel has $s$-wave $(\ell=0)$ character. Hence, we need to consider only even- $\ell$ channels with total projection quantum number $M=-12$ and -16 for ${ }^{168} \mathrm{Er}$ and ${ }^{164} \mathrm{Dy}$, respectively, and inclusion of zero-field solutions up to $J_{\max }=36$ for Dy and 39 for Er is sufficient to reproduce the experimental resonance densities. In Sec. V, we discuss higher-temperature collisions between Er atoms, where $d$-wave $(\ell=2)$ entrance channels must be considered and, hence, spectra at other $M$ values (i.e., $M$ between -14 and -10 for ${ }^{168} \mathrm{Er}$ ) contribute.

\section{Interaction anisotropies}

We first look into the role of interaction anisotropies on the level distribution of the most weakly bound molecular energy levels at zero magnetic field. There are two dominant components to the anisotropy, the dispersion $V_{\Delta C_{6}}(\vec{R})$ and magnetic dipole-dipole $V_{\mathrm{MDD}}(\vec{R})$ contribution. To distinguish the contributions of these two terms, we define

$$
\hat{V}_{a}(\vec{R})=\lambda_{\Delta C_{6}} V_{\Delta C_{6}}(\vec{R})+\lambda_{\mathrm{MDD}} V_{\mathrm{MDD}}(\vec{R}),
$$

with variable strength $\lambda_{\Delta C_{6}}$ and $\lambda_{\mathrm{MDD}}$. We systematically increase the strengths $\lambda_{\Delta C_{6}}$ and $\lambda_{\mathrm{MDD}}$ from zero, where we recover the full physical strength for $\lambda_{\mathrm{MDD}}=\lambda_{\Delta C_{6}}=1$.

For completeness, we note that the dominant tensor operator for the anisotropic dispersion contribution is

$$
V_{\Delta C_{6}}(\vec{R})=\frac{c_{a}}{R^{6}} \sum_{i=1,2} \frac{1}{\sqrt{6}}\left\{3\left(\hat{R} \cdot \vec{\jmath}_{i}\right)\left(\hat{R} \cdot \vec{\jmath}_{i}\right)-\vec{\jmath}_{i} \cdot \vec{\jmath}_{i}\right\}+\cdots,
$$

with strength $c_{a}<0$ found with the methodology discussed in Sec, IVA. Weaker contributions indicated by dots are included in our calculations. Moreover,

$$
V_{\mathrm{MDD}}(\vec{R})=-\frac{\mu_{0}}{4 \pi} \frac{\left(g \mu_{B}\right)^{2}}{R^{3}}\left\{3\left(\hat{R} \cdot \vec{\jmath}_{1}\right)\left(\hat{R} \cdot \vec{J}_{2}\right)-\vec{\jmath}_{1} \cdot \vec{J}_{2}\right\},
$$

where $\mu_{0}$ is the magnetic constant.

Figures 4(b) and 4(d) show the most weakly bound $B=0, J=16$ levels of ${ }^{164} \mathrm{Dy}_{2}$ as a function of anisotropy strength for purely dipolar $\left(\lambda_{\Delta C_{6}}=0\right.$, varying $\left.\lambda_{\mathrm{MDD}}\right)$ and dispersive $\left(\lambda_{\mathrm{MDD}}=0\right.$, varying $\left.\lambda_{\Delta C_{6}}\right)$ anisotropic interaction, respectively. For $\lambda_{\mathrm{MDD}}=\lambda_{\Delta C_{6}}=0$, the binding energies are regularly structured with many near degeneracies. In fact, the corresponding states are rovibrational levels of the isotropic centrifugal potentials $\hat{V}_{i}(R)$ and labeled by $\ell$. In our 3-GHz energy window an $s$-wave channel has at most three bound states, while even $\ell>0$ channels with their centrifugal barriers have fewer $[21,49]$. For small $\lambda_{\Delta C_{6}}$ and $\lambda_{\mathrm{MDD}}$, the degeneracy is lifted and levels shift linearly. The linear dependence for increasing strength of the dipoledipole is approximately valid up to the physical value of $\lambda_{\mathrm{MDD}}=1$. Hence, the dipole-dipole interaction does not lead to our chaotic level distributions. In fact, Fig. 4(a) shows that at $\lambda_{\mathrm{MDD}}=1$ and $\lambda_{\Delta C_{6}}=0$ the NND distribution is Poissonian.

On the other hand, for a relatively small anisotropic dispersion strength $\lambda_{\Delta C_{6}} \approx 0.1$, levels start to avoid each 

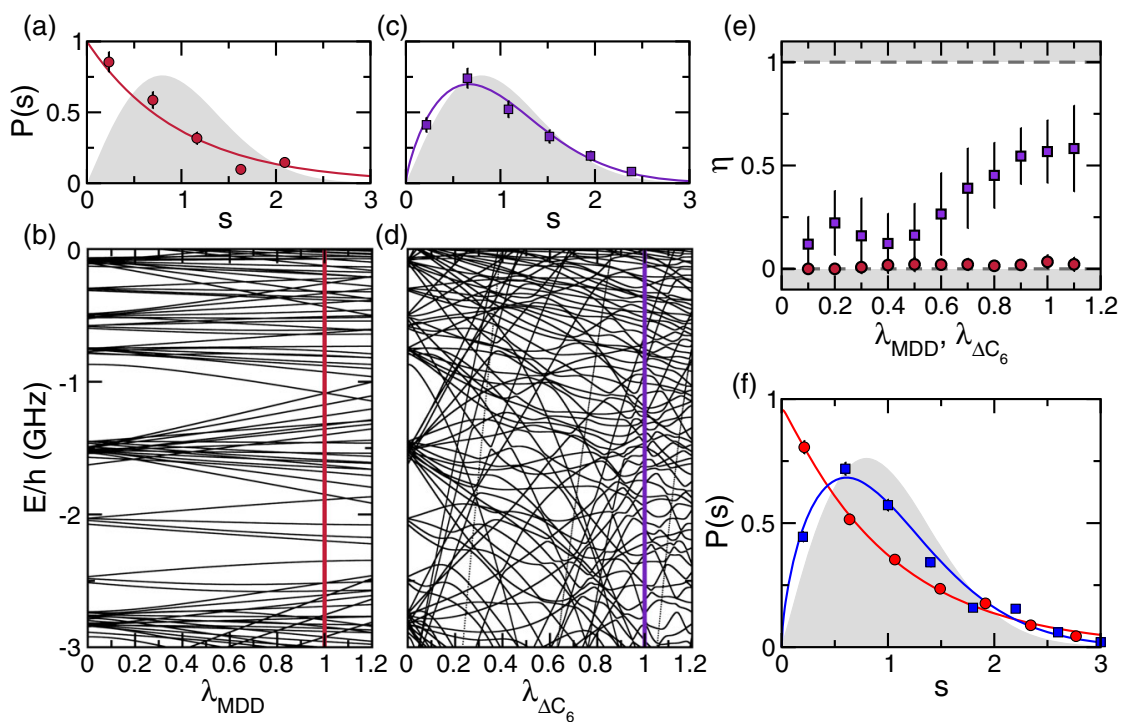

FIG. 4. Interaction-anisotropy-induced chaos of $B=0$ near-threshold bound states. (b) Weakly bound $J=16$ bound-state energies of ${ }^{164} \mathrm{Dy}_{2}$ as a function of the anisotropy scale $\lambda_{\mathrm{MDD}}$ with $\lambda_{\Delta C_{6}}=0$. (a) NNS distribution (red circles) for the $J=16$ bound-state data in (b) at $\lambda_{\mathrm{MDD}}=1$ and $\lambda_{\Delta C_{6}}=0$. The solid red line is a Brody distribution fit to the data and agrees well with a Poisson distribution. (d) Weakly bound $J=16$ bound-state energies of ${ }^{164} \mathrm{Dy}_{2}$ as a function of the anisotropy scale $\lambda_{\Delta C_{6}}$ with $\lambda_{\mathrm{MDD}}=0$. (c) NNS distribution (purple squares) for the $J=16$ bound-state data in (d) at $\lambda_{\mathrm{MDD}}=0$ and $\lambda_{\Delta C_{6}}=1$. The solid purple line is a Brody distribution fit to the data and is close to a Wigner-Dyson distribution. (e) Moving average of the Brody parameter $\eta$ as a function of $\lambda_{\Delta C_{6}}$ (purple squares) or $\lambda_{\text {MDD }}$ (red circles) with bins $\Delta \lambda=0.2$ obtained by fitting the NNS distribution for the $J=16$ bound-state data in (b) and (d) to Brody distributions, respectively. The horizontal lines at $\eta=0$ and 1 correspond to the Brody parameter for a Poisson and Wigner-Dyson distribution, respectively. The $1 \sigma$ error bars combine statistical and fitting uncertainties. (f) The individual- $J$ (blue squares) and combined- $J$ (red circles) NNS distributions $P(s)$ at $\lambda_{\mathrm{MDD}}=\lambda_{\Delta C_{6}}=1$ as a function of the normalized energy spacing $s$. The distributions are derived from $B=0$ bound-state data for $J=16, \ldots, 25$. The gray shaded areas in (a), (b), and (f) indicate the Wigner-Dyson distribution.

other. Starting from $\lambda_{\Delta C_{6}} \approx 0.5$, most avoided crossings are noticeable on the $3-\mathrm{GHz}$ scale of the figure. At the nominal $\lambda_{\Delta C_{6}}=1$, where there are 56 levels with $-3<E / h<0 \mathrm{GHz}$, a significant fraction of the levels have undergone multiple avoided crossings and cannot be described by a single dominant partial wave. The level spacing is chaotic as confirmed by the NND distribution for $\lambda_{\Delta C_{6}}=1$ and $\lambda_{\mathrm{MDD}}=0$ in Fig. 4(c). We compute the weakly bound $J=16$ levels for $\lambda_{\mathrm{MDD}}=\lambda_{\Delta C_{6}}=1$. Visually the level distribution is much the same as the one shown in Fig. 4(d). Similar results have been obtained for ${ }^{168} \mathrm{Er}_{2}$.

Figure 4(e) quantifies the intuition gained from Figs. 4(a)-4(d) by showing the Brody parameter $\eta$ of the $B=0 J=16^{164} \mathrm{Dy}_{2}$ levels as a function of $\lambda_{\Delta C_{6}}$ or $\lambda_{\mathrm{MDD}}$. The Brody parameter is obtained by fitting a Brody distribution to the NNS distribution of the bound state data in Figs. 4(b) and 4(d). For increasing dipole-dipole strength $\lambda_{\mathrm{MDD}}$ and no anisotropic dispersion $\left(\lambda_{\Delta C_{6}}=0\right)$, the parameter is always zero, indicating the prevalence of small level spacings. On the other hand, in the absence of the DDI, increasing $\lambda_{\Delta C_{6}}$ leads to an increasing $\eta$. It evolves from $\eta=0.2$ for $\lambda_{\Delta C_{6}} \lesssim 0.5$ to $\eta=0.7$ for $\lambda_{\Delta C_{6}}=1$, indicating a depopulation of small energy spacings. Note that our systems does not reach a Wigner-Dyson distribution, which corresponds to $\eta=1$.

In Fig. 4(f), we compare two NNS distributions of $B=0$ weakly bound states of ${ }^{164} \mathrm{Dy}_{2}$ obtained for the full anisotropic interaction $\left(\lambda_{\mathrm{MDD}}=\lambda_{\Delta C_{6}}=1\right)$. Both distributions are based on $|E / h|<3 \mathrm{GHz}$ bound states computed for $J=16$ up to 25 . The first so-called individual- $J$ distribution is constructed by averaging the NNS distribution of levels for individual $J$ 's assuming that individual distributions are the same. The second, combined-J NNS distribution, is calculated from a sorted list of all $J=16, \ldots, 25$ levels. Data for $J>25$ are not included as the number of bound states is too small for a reliable determination of the NNS distribution.

The individual- $J$ NNS distribution is non-Poissonian as levels with the same $J$ repel each other. The combined- $J$ distribution, however, follows a Poisson distribution indicating that energies of bound states with different $J$ are uncorrelated. In other words, even though the Hamiltonian, i.e., the set of coupling operators between $\vec{J}_{1}, \vec{J}_{2}$ and $\vec{\ell}$, is the same, differences in the matrix elements and thus coupling strengths between channels lead to uncorrelated eigenenergies. 


\section{Atom scattering in a magnetic field}

The study of the $B=0 \mathrm{G}$ multichannel bound states has shown that interaction anisotropies mix channels with the same $J$, while states with different $J$ are uncorrelated. The Zeeman interaction mixes these molecular levels and leads to the Fano-Feshbach spectrum. Figures 5(a) and 5(b) show example ${ }^{164} \mathrm{Dy}_{2} M=-16$ bound-state spectra as a function of $B$ on two binding-energy and field regions. Similarly, Figs. 5(c) and 5(d) show $M=-12{ }^{168} \mathrm{Er}_{2}$ bound states. In all cases, the full nominal anisotropy $\left(\lambda_{\Delta C_{6}}=1\right.$ and $\lambda_{\mathrm{MDD}}=1$ ) is used. For Dy and Er, channels with $J$ up to 36 and 39 are included, respectively. The figure shows that the Dy level density is higher than that for Er. This simply follows from the larger atomic angular momentum of Dy, leading to a larger number of channels with the same $J-|M|$. We also observe that for both species the level structure in the $0-10 \mathrm{G}$, small field region is qualitatively different from that in the larger field region. For small $B$, the avoided crossings are substantially narrower than for larger $B$. Moreover, at small field the levels cluster, while at larger field they are more uniformly distributed. These changes are a consequence of the linearly increasing Zeeman coupling between vibrational levels with different $J$ 's as a function of $B$.

Figure 6(a) shows effective length $a_{s}(B)$ as a function of $B$. It diverges at every resonance location and is closely related to the scattering length of a zero-energy collision. Our calculations cannot be directly used to define the scattering length as we use a hard-wall potential for $R \geq R_{\max }$. This wall leads to a discrete set of states with positive energy, and using the lowest of these $E_{s}(B)$, we can define the effective length $a_{s}(B)$ shown in the figure by solving for $E_{s}(B)=\hbar^{2} \pi^{2} /\left\{2 \mu_{r}\left[R_{\max }-a_{s}(B)\right]^{2}\right\}$, with $\mu_{r}=m / 2$ and atomic mass $m$ [50].

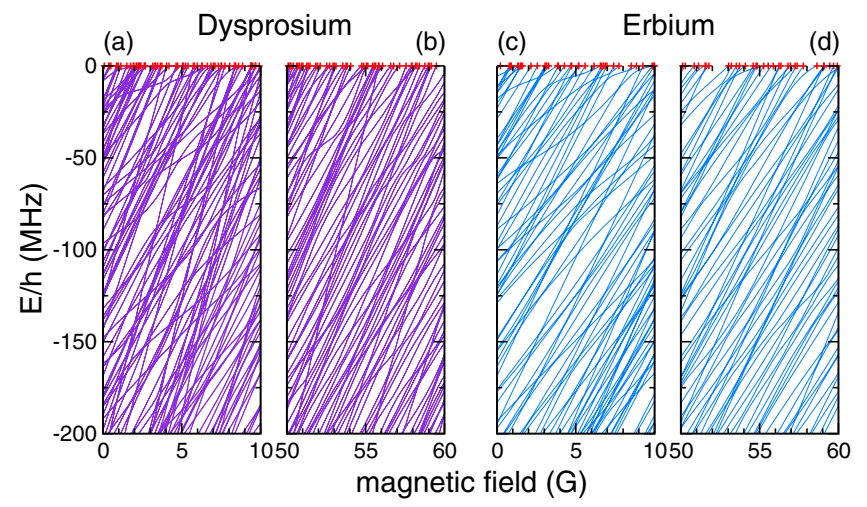

FIG. 5. Theoretical ${ }^{164} \mathrm{Dy}_{2}[(\mathrm{a}),(\mathrm{b})]$ and ${ }^{168} \mathrm{Er}_{2}[(\mathrm{c}),(\mathrm{d})]$ nearthreshold bound states as a function of magnetic field. Calculations have been performed with the full nominal anisotropy. Panels (a) and (c) show the near-threshold region between $B=0$ and $10 \mathrm{G}$, while panels (b) and (d) show the region between $B=50$ and $60 \mathrm{G}$. Red crosses indicate the location of Feshbach resonances.
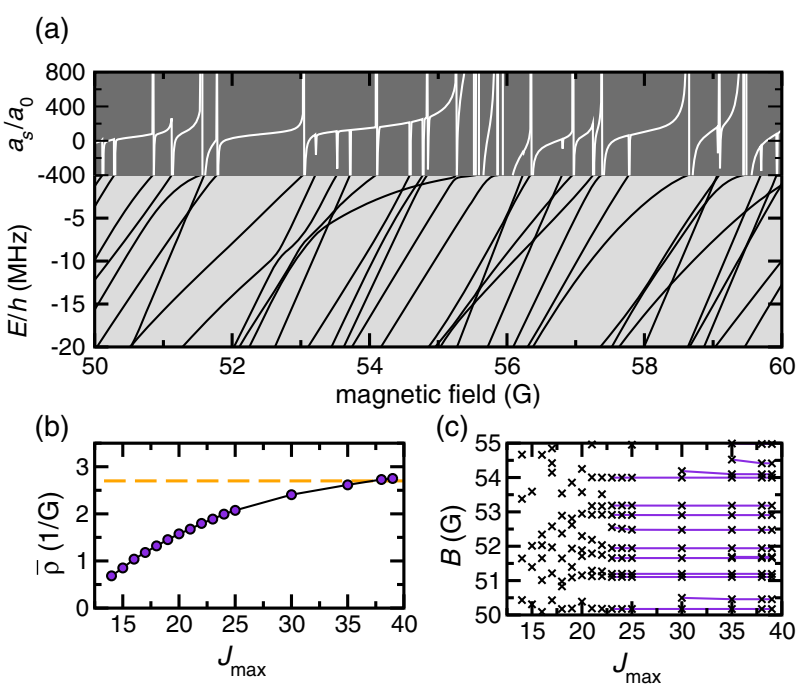

FIG. 6. Theoretical near-threshold bound states and Feshbach resonances in a magnetic field. (a) Near-threshold bound states for $M=-12{ }^{168} \mathrm{Er}_{2}$ for fields between $B=50$ and $60 \mathrm{G}$ (bottom half of image). The top half of the image shows the effective scattering length, defined in the text, as a function of $B$. It is infinite at a resonance location where a bound state has zero energy. Calculations use the physical interaction anisotropies and channel states with $J \leq J_{\max }=39$. (b) Theoretical Feshbachresonance density $\bar{\rho}$ as a function of $J_{\max }$ (purple circles) computed from resonance locations between 0 and $70 \mathrm{G}$. The dashed horizontal line indicates the experimental density for ${ }^{168} \mathrm{Er}_{2}$. (c) Convergence study of resonance locations (crosses) between 50 and $55 \mathrm{G}$ as a function of $J_{\max }$. Purple lines connect resonances when their location has converged.

It is of interest to briefly discuss the convergence properties of our calculations. The data in Figs. 6(a), 5(c), and 5(d) are based on computations with channels with $J$ up to $J_{\max }=39$. Figure 6(b) shows the ${ }^{168} \mathrm{Er}_{2}$ Feshbachresonance density $\bar{\rho}$ as a function of $J_{\max }$. The resonance density increases linearly from $\approx 0.51 / \mathrm{G}$ at $J_{\max }=12$ but then is seen to "saturate" for larger $J_{\max }$. At $J_{\max }=39$ the experimental density is reproduced. In addition, Fig. 6(c) shows the field location of resonances between 50 and $55 \mathrm{G}$ as a function of $J_{\max }$. The resonance locations change significantly for $J_{\max }<22$, but then rapidly converge. This implies strong mixing among bound states with those $J$. On the other hand, the location of resonances that appear for $J \geq 22$ is almost immediately converged indicating weak mixing to smaller $J$ states.

\section{E. Comparison of experiment and coupled-channels model}

In Figs. 2(a) and 2(b), we show the NNS distribution of converged Feshbach-resonance locations based on our multichannel data between $B=0$ and $70 \mathrm{G}$ for ${ }^{164} \mathrm{Dy}_{2}$ with $J_{\max }=36$ and ${ }^{168} \mathrm{Er}_{2}$ with $J_{\max }=39$, respectively. For both species the distribution clearly deviates from a 
Poisson distribution, consistent with the experimental distributions that are also shown. The fitted experimental and coupled-channel Brody parameters agree within their error bars.

The anisotropy parameters $\lambda_{\Delta C_{6}}$ and $\lambda_{\mathrm{MDD}}$ in the coupled-channels calculations and the parameter $\nu_{\text {cpl }}$ in the RMT play analogous roles in the Hamiltonian and in the emergence of chaotic level distributions, even though no explicit quantitative connection exists. This role is most manifest in the Brody parameters of the $B=0 \mathrm{G}$ bound states and that of the Feshbach-resonance spectra for the two models. For ${ }^{168} \mathrm{Er}$ the corresponding Brody parameters from the coupled-channels calculations are $\approx 0.01$ and 0.68 at the physical $\lambda_{\mathrm{MDD}}=\lambda_{\Delta C_{6}}=1$, respectively. Within the RMT model, the small $\eta$ value for the $B=0 \mathrm{G}$ level distribution requires weak coupling $\nu_{\mathrm{cpl}} \ll \epsilon_{d}$ and $\eta_{d} \approx 0$. In contrast, the Brody parameter for the Feshbachresonance spectrum requires $\nu_{\mathrm{cpl}} \approx \epsilon_{d}$ and points at limitations of the current RMT model. Similar conclusions hold for bosonic Dy. Future advanced RMT models might circumvent these limitations by incorporating overlapping, uncoupled chaotic series as is found from our $B=0 \mathrm{G}$ coupled-channels calculations.

We plot the $B$-field-resolved Brody parameter $\eta(B)$ of the theoretical coupled-channels data in Figs. 2(c) and 2(d). A comparison with the experimental $\eta(B)$ shows excellent agreement for ${ }^{164} \mathrm{Dy}$, while the agreement for ${ }^{168} \mathrm{Er}$ is less satisfactory. A possible explanation for the discrepancies in ${ }^{168} \mathrm{Er}$ is the larger bin-to-bin fluctuations as bins contain fewer resonances than for ${ }^{164} \mathrm{Dy}$.

For ${ }^{164}$ Dy the theoretical field-resolved Brody parameter in Fig. 2(c) linearly increases from zero for small $B$ fields and saturates at $\eta(B) \approx 0.5$ for fields larger than $35 \mathrm{G}$, where the size or width of the avoided crossings between weakly bound states is larger. For ${ }^{168} \mathrm{Er}$ in Fig. 2(d) we find a much more rapid increase of $\eta(B)$ at small fields. This is followed by a plateau at $\eta(B) \approx 0.5$ between $B=20$ and $50 \mathrm{G}$, after which $\eta(B) \rightarrow 0.9$ with an uncertainty of 0.2 close to a Wigner distribution. The initial rise of $\eta(B)$ for both atomic species is a consequence of weakly bound vibrational levels, uncoupled and randomly distributed when $B=0 \mathrm{G}$, that start to repel each other as the Zeeman interaction increases in strength for increasing $B$. The plateau at $\eta(B) \approx 0.5$ and the sudden increase of $\eta(B)$ to one for ${ }^{168} \mathrm{Er}$ have no simple explanation and are determined by the not-fully-explored complex interplay between the Zeeman and anisotropic interatomic interactions. It does, however, indicate that Wigner's assumptions on ensembles of Hamiltonians do not hold for fields below $50 \mathrm{G}$.

\section{TEMPERATURE DEPENDENCE OF THE RESONANCE DENSITY}

We now describe the origin of the strong temperature dependence of some of the resonances in our atom-loss spectra and thus explain the accompanying increase of the resonance density. Here, atom loss is solely due to threebody recombination, where three ultracold atoms collide to form a diatomic molecule and an atom that are both lost from the atom trap. Figure 7(a) shows atom-loss spectra for one such resonance for ${ }^{168} \mathrm{Er}$ at four temperatures below $2 \mu \mathrm{K}$. Atom loss, indeed, is larger for larger temperatures, but we also observe a broadening of the $B$-field width and a shift of the maximum loss position to larger $B$ fields.
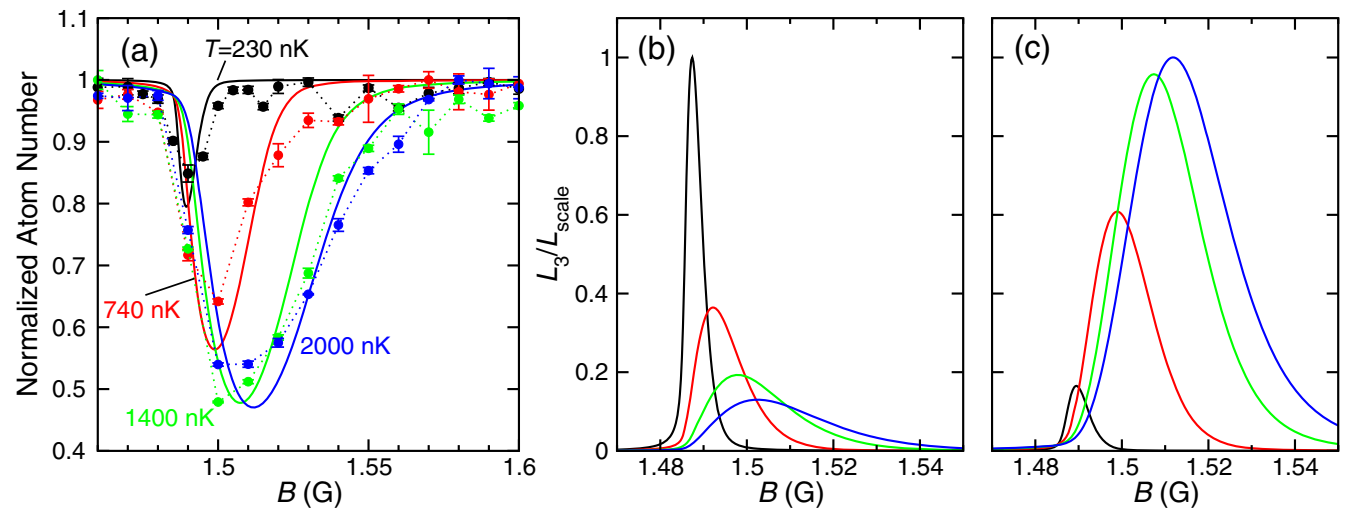

FIG. 7. Line shapes for a strongly temperature-dependent ${ }^{168} \mathrm{Er}$ Feshbach resonance near $B=1.48$ G. Panel (a) shows experimental data (markers with error bars) as a function of $B$ of the remaining atom number divided by the atom number away from resonance measured $400 \mathrm{~ms}$ after initial preparation. Black, red, green, and blue markers correspond to data for temperatures $T=230,740,1400$, and $2000 \mathrm{nK}$, respectively. Dashed lines connecting the markers guide the eye. Solid lines are theoretical line shapes of the remaining atom number based on the $d$-wave $(\mathcal{N}=2$ ) recombination rates shown in (c). Panels (b) and (c) show simulated three-body recombination rates for the same four temperatures assuming three-body entrance $s$ - $(\mathcal{N}=0)$ and $d$-wave $(\mathcal{N}=2)$ scattering, respectively. Curves are based on a thermally averaged line shape discussed in the text. Recombination rates are scaled such that the largest value in each panel is one. For both panels, $\mu=3.1 \mu_{B}$ and $\Gamma_{\mathrm{br}} / k_{B}=250 \mathrm{nK}$, while $\Gamma(E) / k_{B}=0.2\left(E / E_{\mathrm{ref}}\right)^{2} \mathrm{nK}$ in (b) and $\Gamma(E) / k_{B}=0.1\left(E / E_{\mathrm{ref}}\right)^{4} \mathrm{nK}$ in $(\mathrm{c})$, where $E_{\mathrm{ref}} / k_{B}=1000 \mathrm{nK}$. 
Resonances with a weak temperature dependence show none of these behaviors.

We show with an intuitive resonant "trimer" model that a strongly temperature-dependent resonance is due to scattering processes with entrance $d$-wave channels even though the two-body $d$-wave centrifugal barrier, $V_{b} / k_{B}=250 \mu \mathrm{K}$, is 100 times larger than our highest temperature, where $k_{B}$ is the Boltzmann constant. The difference in the power-law Wigner-threshold behavior of the recombination rate with collision energy for $s$ - and $d$-wave entrance-channel collisions can explain our observations.

Three-body recombination has been extensively studied in the context of Efimov physics [51-54]. We follow Refs. [55-57] and start from a coupled-channels description in the (mass-scaled) hyperradius $\rho$, which describes the size of the three-atomic system, and basis functions in the five other hyperspherical coordinates that are $\rho$-dependent eigenstates of the squared "grand-angular-momentum operator." Similar to the coupled-channels description for two atoms, there are entrance, open, and closed channels. The collision starts in one of the entrance channels with atoms in the energetically lowest Zeeman state and relative threebody kinetic energy $E_{3}$, the dimer plus atom are the open channels, and bound states in closed channels can lead to resonances. These closed channels dissociate to three freeatom states with at least one atom in a Zeeman level with higher internal energy. The bound states are resonant trimer states giving us our name for the model. It should, however, be realized that their origin lies in bound states of pairs of atoms and that the resonant state is better thought of as a pair bound state that hops from pair to pair. We define $E_{3} \equiv \hbar^{3} k_{3}^{2} /\left(2 \mu_{3}\right) \equiv \mu_{3} v_{3}^{2} / 2$ with the three-body reduced mass $\mu_{3}=m / \sqrt{3}$, where $k_{3}$ and $v_{3}$ are the relative wave vector and velocity, respectively.

The potentials in the entrance channels have long-range repulsive centrifugal potentials, governed by the asymptotic behavior of the grand-angular-momentum operator, and depend on the relative orbital angular momentum $\overrightarrow{\mathcal{N}}$ of the three atoms. In fact, the centrifugal potentials are $\hbar^{2}(\lambda+3 / 2)(\lambda+5 / 2) /\left(2 \mu_{3} \rho^{2}\right)$ with non-negative integer quantum number $\lambda$ [55]. For $\mathcal{N}=0$, the least repulsive potential has $\lambda=0$, while that for $\mathcal{N}=2$ has $\lambda=2$.

For an isolated trimer resonance in a closed channel coupled to both entrance and other open channels, we can apply the resonance theories by Fano and Feshbach and derive that the recombination rate coefficient at collision energy $E_{3}$ and entrance channel with quantum number $\lambda$ is given by $L_{3}\left(E_{3}, B\right)=v_{3} \sigma\left(E_{3}, B\right)$, where the cross section $\sigma\left(E_{3}, B\right)=(2 \mathcal{N}+1) 192 \pi^{2}\left|S\left(E_{3}, B\right)\right|^{2} / k_{3}^{5}$ and

$$
\left|S\left(E_{3}, B\right)\right|^{2}=\frac{\Gamma\left(E_{3}\right) \Gamma_{\mathrm{br}}}{\left[E_{3}-\mu\left(B-B_{0}\right)\right]^{2}+\left[\Gamma_{\mathrm{tot}}\left(E_{3}\right) / 2\right]^{2}}
$$

is a resonant expression for the square of a dimensionless $S$-matrix element, where $B_{0}$ is the trimer resonance location and $\mu$ is the magnetic moment of the resonant trimer relative to that of the entrance channel. The definition for $\left|S\left(E_{3}, B\right)\right|^{2}$ also contains the entrance-channel energy width $\Gamma\left(E_{3}\right)=A_{\lambda} E_{3}^{\lambda+2}$ to the trimer resonance with a characteristic power-law energy dependence that reflects the threshold behavior of the scattering solutions in the centrifugal potentials. The energy width $\Gamma_{\mathrm{br}}$ determines the decay or breakup rate of the resonance into the fast atom and dimer pair and is independent of $E_{3}$. Finally, $\Gamma_{\text {tot }}\left(E_{3}\right)=\Gamma\left(E_{3}\right)+\Gamma_{\mathrm{br}}$. For simplicity, we assume that nonresonant, direct recombination from the entrance to open channels is weak. We also note that for $\mathcal{N}=0$ and $\lambda=0, L_{3}\left(E_{3}, B\right)$ approaches a finite constant for $E_{3} \rightarrow 0$ as expected.

In our experiments we have thermal samples of Er and we require the thermally averaged rate coefficient

$$
L_{3}(T, B)=\frac{1}{Z} \int_{0}^{\infty} E^{2} d E L_{3}(E, B) e^{-E / k T}
$$

and normalization $Z=\int_{0}^{\infty} E^{2} d E e^{-E / k T}=2(k T)^{3}$. In order to increase the signal-to-noise ratio, we allow a significant fraction of atoms to be lost [see Fig. 7(c)], which, assuming a homogeneous sample, can be modeled by the rate equation $d n(t) / d t=-3 L_{3}(T, B) n^{3}(t)$ for atom density $n(t)$ [53] with solution

$$
N\left(t_{h}, B\right)=\frac{N_{0}}{\sqrt{1+6 L_{3}(T, B) n_{0}^{2} t_{h}}},
$$

where $N\left(t_{h}, B\right)$ is the remaining atom number after hold time $t_{h}, N_{0}$ is the initial atom number, and $n_{0}$ is the initial density. This nonlinear time evolution adds additional broadening to the lines.

Figures 7(b) and 7(c) show our model event rates $L_{3}(T, B)$ as a function of $B$ for $\mathcal{N}=0, \lambda=0$ and $\mathcal{N}=2, \lambda=2$, respectively. Curves are for the same four temperatures as in Fig. 7(a). A comparison of Figs. 7(b) and 7(c) shows a striking difference. The strongest features in Fig. 7(b) are for the smallest temperatures, while those in Fig. 7(c) are for the largest temperatures. This behavior naturally follows from an approximation of the integrant in $L_{3}(T, B)$ under the conditions $k T \gg \Gamma_{\text {br }} \gg \Gamma(E)$ [58]. In this limit the Lorentzian is sharply peaked around $E_{3}=$ $\mu\left(B-B_{0}\right)$ for $B>B_{0}$, and after some algebra it follows that $L_{3}(T, B)$ as a function of $B$ has a maximum value proportional to $(k T)^{\lambda-1}$ located at $B=B_{0}+(\lambda+2) k T / \mu$. Consequently, for $\lambda=0$ and 2 the maximum loss rate decreases and increases with $T$, respectively. Even for less restrictive parameter values as used in Fig. 7 this trend remains.

Our experimental data have a temperature trend as in Fig. 7(c). In fact, Fig. 7(a) compares our experimental loss data with model $N\left(t_{h}, B\right)$ for $\mathcal{N}=2, \lambda=2$ using the same parameters as in Fig. $7(\mathrm{c})$ and requiring a $\approx 50 \%$ 
maximum atom loss as in the experiment. It is worth noting that, from our theoretical calculations, the magnetic-field width of $L_{3}(T, B)$ is noticeably smaller than that for $N\left(t_{h}, B\right)$, indicating that the finite hold time does indeed lead to broadening. The agreement of the experimental data and the prediction of our model for the losses is satisfactory for all four temperatures given the limitations and approximations within our modeling. We conclude that our strongly $T$-dependent resonances correspond to $d$-wave or more precisely $\mathcal{N}=2$ entrance-channel collisions. Note that we have not observed any resonances with temperature dependence similar to Fig. 7(b) in our spectra. In the case of resonances with a three-body $s$-wave entrance channel, which would correspond to such a dependence, we infer that the loss spectra are saturated. This will be subject for future investigations.

As a corollary, this implies that for two colliding atoms, as described in Sec. IV, temperature-dependent resonances are due to collisions with entrance $d$ waves for which there are multiple allowed values of the total angular projection quantum number $M$. Here, $M=-14$ to -10 for bosonic $\mathrm{Er}$ and $M=-18$ to -14 for bosonic Dy. Numerical computations, not presented here, show that their zero-field bound states and thus resonance locations are again uncorrelated and random.

\section{CONCLUSION}

In summary, we experimentally and theoretically study the resonant scattering of ultracold Er and Dy atoms in a magnetic field. We show that chaotic scattering as witnessed by chaotic nearest-neighbor spacings between Feshbach-resonance locations emerges due to the anisotropy in the molecular dispersion.

Our study also reveals several unique features of colliding magnetic lanthanides that have not been observed in any other ultracold atomic system. These lanthanides are characterized by their exceptionally large electron orbital angular momentum, which leads to large anisotropic dispersion interactions between these atoms. Our theoretical estimate shows that in both Er and Dy collisions the ratio of anisotropic to isotropic dispersion interaction $\Delta C_{6} / C_{6}$ is about $10 \%$. This anisotropy leads to significant splittings among the 48 and 81 gerade short-range potentials that dissociate to the ground-state atomic limits of Er and Dy, respectively. We show that each potential has its own rovibrational structure, which by Coriolis forces and the Zeeman interaction interacts with that of other potentials, creating a dense distribution of levels near the threshold and initiating chaos. In fact, we find a very large number of partial waves contributing to the creation of Fano-Feshbach resonances.

On the other hand, if we just consider the anisotropy from the magnetic dipole-dipole interaction alone, our coupled-channel calculations indicate that chaos in the level distribution does not appear. The strength of the dipole-dipole interaction is too small. In addition, we show that the NNS distributions for Dy and Er are very similar, as can be expected from their similar $\Delta C_{6} / C_{6}$ ratio. The difference in their magnetic moment plays only a small role. This further confirms that chaos is due to the anisotropic dispersion interaction.

The distribution of Feshbach resonances of ultracold ground-state alkali-metal, alkaline-earth, $\mathrm{Yb}$, and $\mathrm{Cr}$ atoms, as experimental studies have shown, is not chaotic. This is because these atoms have a zero electron orbital angular momentum and, hence, only an isotropic dispersion interaction. Even though alkali-metal and $\mathrm{Cr}$ atoms have a nonzero magnetic moment of $1 \mu_{B}$ and $6 \mu_{B}$, respectively, these moments do not lead to chaos. We would expect that other magnetic lanthanides and actinides with nonzero orbital angular momentum will exhibit chaotic properties in their collisions. In addition, collisions between mixed species, such as magnetic lanthanides and alkali metals, like $\mathrm{K}+\mathrm{Dy}$ or $\mathrm{Na}+\mathrm{Er}$, might be susceptible to chaos. A first theoretical analysis for $\mathrm{Li}+\mathrm{Er}$ [59], however, estimates a small $2 \%$ dispersion anisotropy and no chaos is predicted.

Another interesting property of magnetic lanthanide gases is the extreme sensitivity of the atom-loss spectra and, in essence, three-body recombination to the temperature. This phenomenon was first observed in Ref. [22] for loss spectra of Dy. The number of Dy resonances increases by $50 \%$ when the temperature is increased from 420 to $800 \mathrm{nK}$. Here, we observe a $25 \%$ increase in the $\mathrm{Er}$ resonance density when the temperature rises from 250 to $1400 \mathrm{nK}$. We show by a comparison of resonance profiles taken at several temperatures and predictions of a theoretical model of three-body recombination via the formation of a trimer, or, more precisely, of a shared pair bound state, that the origin of the temperature-dependent resonances lies in the "partial wave" of the three-atom entrance channel. Entrance channels with zero and nonzero total orbital angular momentum $\mathcal{N}$ lead to line shapes with a different temperature behavior. Those with $\mathcal{N}=0$ or "s-wave" entrance channels have sharply decreasing recombination rates with temperature, whereas those with $\mathcal{N}=2$ or " $d$-wave" entrance channels have an increasing recombination rate. Temperature-sensitive resonances can be explained only by " $d$-wave" collisions. It is worth noting that for alkali-metal-atom collisions a number of entrance-channel $p$-wave resonances have been observed (see, for example, Ref. [60] for cesium). Analysis of the temperature-dependent rate coefficient, however, was not performed.

\section{ACKNOWLEDGMENTS}

The Stuttgart group thanks Axel Griesmaier for the support at the early stage of the experiment. The Dy work is supported by the German Research Foundation (DFG) within SFB/TRR21. H. K. acknowledges support by the 
"Studienstiftung des deutschen Volkes." The Er work is supported by the Austrian Ministry of Science and Research (BMWF) and the Austrian Science Fund (FWF) through a START grant under Project No. Y479N20 and by the European Research Council under Project No. 259435. K. A. was supported within the Lise-Meitner program of the FWF. L. C. acknowledges support by the FWF through SFB FoQuS. Work at Temple University is supported by the AFOSR Grant No. FA9550-14-1-0321 and the NSF Grant No. PHY-1308573. The work at JQI is supported by the NSF Grant No. PHY-1506343.

[1] M. A. Baranov, M. Dalmonte, G. Pupillo, and P. Zoller, Condensed Matter Theory of Dipolar Quantum Gases, Chem. Rev. 112, 5012 (2012).

[2] G. Quemener and P. S. Julienne, Ultracold Molecules under Control!, Chem. Rev. 112, 4949 (2012).

[3] M. Saffman, T. G. Walker, and K. Mølmer, Quantum Information with Rydberg Atoms, Rev. Mod. Phys. 82, 2313 (2010).

[4] T. Lahaye, C. Menotti, L. Santos, M. Lewenstein, and T. Pfau, The Physics of Dipolar Bosonic Quantum Gases, Rep. Prog. Phys. 72, 126401 (2009).

[5] T. Lahaye, J. Metz, B. Fröhlich, T. Koch, M. Meister, A. Griesmaier, T. Pfau, H. Saito, Y. Kawaguchi, and M. Ueda, $d$-Wave Collapse and Explosion of a Dipolar Bose-Einstein Condensate, Phys. Rev. Lett. 101, 080401 (2008).

[6] K. Aikawa, S. Baier, A. Frisch, M. Mark, C. Ravensbergen, and F. Ferlaino, Observation of Fermi Surface Deformation in a Dipolar Quantum Gas, Science 345, 1484 (2014).

[7] M. H. G. de Miranda, A. Chotia, B. Neyenhuis, D. Wang, G. Quéméner, S. Ospelkaus, J. L. Bohn, J. Ye, and D. S. Jin, Controlling the Quantum Stereodynamics of Ultracold Bimolecular Reactions, Nat. Phys. 7, 502 (2011).

[8] J. L. Bohn, M. Cavagnero, and C. Ticknor, Quasi-Universal Dipolar Scattering in Cold and Ultracold Gases, New J. Phys. 11, 055039 (2009).

[9] Y. Wang, J. P. D'Incao, and C. H. Greene, Universal ThreeBody Physics for Fermionic Dipoles, Phys. Rev. Lett. 107, 233201 (2011).

[10] L. Santos, G. V. Shlyapnikov, and M. Lewenstein, Roton-Maxon Spectrum and Stability of Trapped Dipolar Bose-Einstein Condensates, Phys. Rev. Lett. 90, 250403 (2003).

[11] S. Ronen, D. C. E. Bortolotti, and J. L. Bohn, Radial and Angular Rotons in Trapped Dipolar Gases, Phys. Rev. Lett. 98, 030406 (2007).

[12] I. Tikhonenkov, B. A. Malomed, and A. Vardi, Anisotropic Solitons in Dipolar Bose-Einstein Condensates, Phys. Rev. Lett. 100, 090406 (2008).

[13] I. Danshita and C. A. R. Sá de Melo, Stability of Superfluid and Supersolid Phases of Dipolar Bosons in Optical Lattices, Phys. Rev. Lett. 103, 225301 (2009).

[14] K. Aikawa, A. Frisch, M. Mark, S. Baier, A. Rietzler, R. Grimm, and F. Ferlaino, Bose-Einstein Condensation of Erbium, Phys. Rev. Lett. 108, 210401 (2012).
[15] K. Aikawa, A. Frisch, M. Mark, S. Baier, R. Grimm, and F. Ferlaino, Reaching Fermi Degeneracy via Universal Dipolar Scattering, Phys. Rev. Lett. 112, 010404 (2014).

[16] M. Lu, N. Q. Burdick, S. H. Youn, and B. L. Lev, Strongly Dipolar Bose-Einstein Condensate of Dysprosium, Phys. Rev. Lett. 107, 190401 (2011).

[17] M. Lu, N. Q. Burdick, and B. L. Lev, Quantum Degenerate Dipolar Fermi Gas, Phys. Rev. Lett. 108, 215301 (2012).

[18] A. Petrov, E. Tiesinga, and S. Kotochigova, AnisotropyInduced Feshbach Resonances in a Quantum Dipolar Gas of Highly Magnetic Atoms, Phys. Rev. Lett. 109, 103002 (2012).

[19] J. Miao, J. Hostetter, G. Stratis, and M. Saffman, Magnetooptical Trapping of Holmium Atoms, Phys. Rev. A 89, 041401 (2014).

[20] D. Sukachev, A. Sokolov, K. Chebakov, A. Akimov, S. Kanorsky, N. Kolachevsky, and V. Sorokin, Magneto-optical Trap for Thulium Atoms, Phys. Rev. A 82, 011405 (2010).

[21] A. Frisch, M. Mark, K. Aikawa, F. Ferlaino, J. L. Bohn, C. Makrides, A. Petrov, and S. Kotochigova, Quantum Chaos in Ultracold Collisions of Gas-Phase Erbium Atoms, Nature (London) 507, 475 (2014).

[22] K. Baumann, N. Q. Burdick, M. Lu, and B. L. Lev, Observation of Low-Field Fano-Feshbach Resonances in Ultracold Gases of Dysprosium, Phys. Rev. A 89, 020701 (2014).

[23] R. Blümel and W. P. Reinhardt, Chaos in Atomic Physics (Cambridge University Press, Cambridge, England, 1997).

[24] V. V. Flambaum, A. A. Gribakina, and G. F. Gribakin, Statistics of Electromagnetic Transitions as a Signature of Chaos in Many-Electron Atoms, Phys. Rev. A 58, 230 (1998).

[25] V. V. Flambaum, M. G. Kozlov, and G. F. Gribakin, Coherent and Stochastic Contributions of Compound Resonances in Atomic Processes: Electron Recombination, Photoionization, and Scattering, Phys. Rev. A 91, 052704 (2015).

[26] O. Agam, B. L. Altshuler, and A. V. Andreev, Spectral Statistics: From Disordered to Chaotic Systems, Phys. Rev. Lett. 75, 4389 (1995).

[27] T. Zelevinsky, S. Kotochigova, and J. Ye, Precision Test of Mass-Ratio Variations with Lattice-Confined Ultracold Molecules, Phys. Rev. Lett. 100, 043201 (2008).

[28] H. I. Liou, H.S. Camarda, and F. Rahn, Application of Statistical Tests for Single-Level Populations to NeutronResonance-Spectroscopy Data, Phys. Rev. C 5, 1002 (1972).

[29] H. I. Liou, H. S. Camarda, S. Wynchank, M. Slagowitz, G. Hacken, F. Rahn, and J. Rainwater, Neutron-Resonance Spectroscopy. VIII. The Separated Isotopes of Erbium: Evidence for Dyson's Theory Concerning Level Spacings, Phys. Rev. C 5, 974 (1972).

[30] V. Zelevinsky, B. A. Brown, N. Frazier, and M. Horoi, The Nuclear Shell Model as a Testing Ground for Many-Body Quantum Chaos, Phys. Rep. 276, 85 (1996).

[31] N. Frazier, B. A. Brown, and V. Zelevinsky, Strength Functions and Spreading Widths of Simple Shell Model Configurations, Phys. Rev. C 54, 1665 (1996).

[32] A. Molinari and H. A. Weidenmüller, Nuclear Masses, Chaos, and the Residual Interaction, Phys. Lett. B 637, 48 (2006). 
[33] H. A. Weidenmüller and G. E. Mitchell, Random Matrices and Chaos in Nuclear Physics: Nuclear Structure, Rev. Mod. Phys. 81, 539 (2009).

[34] T. Maier, H. Kadau, M. Schmitt, A. Griesmaier, and T. Pfau, Narrow-Line Magneto-optical Trap for Dysprosium Atoms, Opt. Lett. 39, 3138 (2014).

[35] T. Lauber, J. Küber, O. Wille, and G. Birkl, Optimized BoseEinstein-Condensate Production in a Dipole Trap based on a 1070-nm Multifrequency Laser: Influence of Enhanced Two-Body Loss on the Evaporation Process, Phys. Rev. A 84, 043641 (2011).

[36] C. Chin, R. Grimm, P. Julienne, and E. Tiesinga, Feshbach Resonances in Ultracold Gases, Rev. Mod. Phys. 82, 1225 (2010).

[37] H. A. Weidenmüller and G. E. Mitchell, Random Matrices and Chaos in Nuclear Physics: Nuclear Structure, Rev. Mod. Phys. 81, 539 (2009).

[38] T. A. Brody, J. Flores, J. B. French, P. A. Mello, A. Pandey, and S. S. M. Wong, Random-Matrix Physics: Spectrum and Strength Fluctuations, Rev. Mod. Phys. 53, 385 (1981).

[39] F. J. Dyson, Statistical Theory of the Energy Levels of Complex Systems. I, J. Math. Phys. (N.Y.) 3, 140 (1962).

[40] A. Petrov, E. Tiesinga, and S. Kotochigova, AnisotropyInduced Feshbach Resonances in a Quantum Dipolar Gas of Highly Magnetic Atoms, Phys. Rev. Lett. 109, 103002 (2012).

[41] M. E. Wickliffe, J. E. Lawler, and G. Nave, Atomic Transition Probabilities for Dy I and Dy II, J. Quant. Spectrosc. Radiat. Transfer 66, 363 (2000).

[42] J. J. Curry, E. A. Den Hartog, and J. E. Lawler, Radiative Lifetimes of Dy I and Dy II, J. Opt. Soc. Am. B 14, 2788 (1997).

[43] V. N. Gorshkov, V. A. Komarovskii, A. L. Oserovich, N. P. Penkin, and R. Khefferlin, Lifetimes of Dy I and Dy II Excited Levels. Oscillator Strengths of Dy I Spectral Lines, Opt. Spectrosc. 48, 362 (1980).

[44] V. A. Dzuba, V. V. Flambaum, and B. L. Lev, Dynamic Polarizabilities and Magic Wavelengths for Dysprosium, Phys. Rev. A 83, 032502 (2011).

[45] C. J. Williams, E. Tiesinga, and P. S. Julienne, Hyperfine Structure of the $\mathrm{Na}_{2} \mathrm{O}_{g}^{-}$Long-Range Molecular State, Phys. Rev. A 53, R1939 (1996).

[46] E. Tiesinga, C. J. Williams, and P. S. Julienne, Photoassociative Spectroscopy of Highly Excited Vibrational Levels of Alkali-Metal Dimers: Green-Function Approach for Eigenvalue Solvers, Phys. Rev. A 57, 4257 (1998).
[47] V. Kokoouline, O. Dulieu, and F. Masnou-Seeuws, Theoretical Treatment of Channel Mixing in Excited $\mathrm{Rb}_{2}$ and $\mathrm{Cs}_{2}$ Ultracold Molecules: Perturbations in $0_{u}^{+}$Photoassociation and Fluorescence Spectra, Phys. Rev. A 62 , 022504 (2000).

[48] D. T. Colbert and W. H. Miller, A Novel Discrete Variable Representation for Quantum-Mechanical Reactive Scattering via the S-Matrix Kohn Method, J. Chem. Phys. 96, 1982 (1992).

[49] B. Gao, E. Tiesinga, C. J. Williams, and P. S. Julienne, Multichannel Quantum-Defect Theory for Slow Atomic Collisions, Phys. Rev. A 72, 042719 (2005).

[50] K. Burnett, P. S. Julienne, P. D. Lett, E. Tiesinga, and C. J. Williams, Quantum Encounters of the Cold Kind, Nature (London) 416, 225 (2002).

[51] D.S. Petrov, Three-Boson Problem near a Narrow Feshbach Resonance, Phys. Rev. Lett. 93, 143201 (2004).

[52] T. Kraemer, M. Mark, P. Waldburger, J. G. Danzl, C. Chin, B. Engeser, A. D. Lange, K. Pilch, A. Jaakkola, H. C. Nägerl, and R. Grimm, Evidence for Efimov Quantum States in an Ultracold Gas of Caesium Atoms, Nature (London) 440, 315 (2006).

[53] E. Braaten and H.-W. Hammer, Universality in Few-Body Systems with Large Scattering Length, Phys. Rep. 428, 259 (2006).

[54] P. Massignan and H. T. C. Stoof, Efimov States near a Feshbach Resonance, Phys. Rev. A 78, 030701 (2008).

[55] H. Suno, B. D. Esry, C. H. Greene, and J. P. Burke, ThreeBody Recombination of Cold Helium Atoms, Phys. Rev. A 65, 042725 (2002).

[56] J. Wang, J. P. D'Incao, and C. H. Greene, Numerical Study of Three-Body Recombination for Systems with Many Bound States, Phys. Rev. A 84, 052721 (2011).

[57] J. Wang, J. P. D'Incao, Y. Wang, and C. H. Greene, Universal Three-Body Recombination via Resonant d-Wave Interactions, Phys. Rev. A 86, 062511 (2012).

[58] K. M. Jones, P. D. Lett, E. Tiesinga, and P. S. Julienne, Fitting Line Shapes in Photoassociation Spectroscopy of Ultracold Atoms: A Useful Approximation, Phys. Rev. A 61, 012501 (1999).

[59] M. L. González-Martínez and P. S. Zuchowski, Magnetically Tunable Feshbach Resonances in Li + Er, Phys. Rev. A 92, 022708 (2015).

[60] C. Chin, V. Vuleticć, A. J. Kerman, S. Chu, E. Tiesinga, P. J. Leo, and C. J. Williams, Precision Feshbach Spectroscopy of Ultracold $\mathrm{Cs}_{2}$, Phys. Rev. A 70, 032701 (2004). 